\title{
Global Measles and Rubella Laboratory Network Support for Elimination Goals, 2010-2015
}

\begin{abstract}
Mick N. Mulders, PhD ${ }^{1}$; Paul A. Rota, PhD²; Joseph P. Icenogle, PhD²; Kevin E. Brown, MD³; Makoto Takeda, MD, PhD; Gloria J. Rey, MSc ${ }^{5}$; Myriam C. Ben Mamou, MD6; Annick R.G.A. Dosseh, PhD; Charles R. Byabamazima, MD ${ }^{8}$; Hinda J. Ahmed, PhD'; Sirima Pattamadilok, MSc ${ }^{10}$; Yan Zhang, MD ${ }^{11}$; Marta Gacic-Dobo, MSc ${ }^{1}$; Peter M. Strebel, MBChB ${ }^{1}$; James L. Goodson, $\mathrm{MPH}^{12}$
\end{abstract}

In 2012, the World Health Assembly endorsed the Global Vaccine Action Plan (GVAP)* with the objective to eliminate measles and rubella in five World Health Organization (WHO) regions by 2020. In September 2013, countries in all six WHO regions had established measles elimination goals, and additional goals for elimination of rubella and congenital rubella syndrome were established in three regions (1). Capacity for surveillance, including laboratory confirmation, is fundamental to monitoring and verifying elimination. The 2012-2020 Global Measles and Rubella Strategic Plan of the Measles and Rubella Initiative ${ }^{\dagger}$ calls for effective case-based surveillance with laboratory testing for case confirmation (2). In 2000, the WHO Global Measles and Rubella Laboratory Network (GMRLN) was established to provide high quality laboratory support for surveillance (3). The GMRLN is the largest globally coordinated laboratory network, with 703 laboratories supporting surveillance in 191 countries. During 2010-2015, 742,187 serum specimens were tested, and 27,832 viral sequences were reported globally. Expansion of the capacity of the GMRLN will support measles and rubella elimination efforts as well as surveillance for other vaccine-preventable diseases (VPDs), including rotavirus, and for emerging pathogens of public health concern.

\section{GMRLN Structure, Coordination, and Responsibilities}

The GMRLN has a multitiered structure based on the design of the WHO Global Polio Laboratory Network. ${ }^{\S}$ In 2015, measles surveillance in $191^{\circ}$ countries was supported by 703 GMRLN laboratories based in 165 countries, including

\footnotetext{
* The Global Vaccine Action Plan is the implementation plan of the Decade of Vaccines, a collaboration between WHO, UNICEF, the Bill and Melinda Gates Foundation, Gavi, the Vaccine Alliance, the U.S. National Institute of Allergy and Infectious Diseases, the African Leaders Malaria Alliance, and others, to extend the full benefit of immunization to all persons by 2020 and beyond. http://www.who.int/immunization/global_vaccine_action_plan/en. http:// apps.who.int/gb/ebwha/pdf_files/wha65/a65_22-en.pdf.

$\dagger$ The Measles and Rubella Initiative is a partnership established in 2001 as the Measles Initiative, led by the American Red Cross, CDC, the United Nations Foundation, UNICEF, and WHO. http://measlesrubellainitiative.org/.

$\$$ http://www.polioeradication.org/Dataandmonitoring/Surveillance/ GlobalPolioLaboratoryNetwork.aspx.

SCountries without access to standardized quality-controlled testing by the WHO Measles and Rubella Laboratory Network in 2015 were Cape Verde, Sao Tome and Principe, and Seychelles.
}

506 subnational, 180 national, 14 regional reference, and three global specialized laboratories, with some laboratories having more than one designation. GMRLN is led by a global coordinator at WHO headquarters, and each region has at least one regional laboratory coordinator. The national and subnational laboratories perform the first-line laboratory testing required for case confirmation and are closely linked with the national immunization programs. Regional reference laboratories support national laboratories by providing confirmatory testing, proficiency testing, training, and support for genetic characterization of circulating wild-type viruses. The global specialized laboratories contribute to the standardization of procedures and protocols, the development and validation of novel methods, and the provision of crucial reagents, supplies, and training.

GMRLN laboratories play a critical role in the process of verification of elimination, which relies on high quality case-based surveillance. Laboratory performance indicators monitored by WHO include 1) the rate of discarded nonmeasles/nonrubella cases** at the national level (target $\geq 2$ per 100,000 population), 2) the proportion of suspected cases with a serum sample obtained for laboratory confirmation, 3) the proportion of laboratory-confirmed chains of transmission with an adequate sample obtained for virus detection (i.e., adequacy of virologic surveillance), and 4) the proportion of serologic results reported within 4 days of specimen receipt in the laboratory (4).

\section{Laboratory Testing}

The annual number of measles cases identified from casebased and aggregate surveillance systems ${ }^{\dagger \dagger}$ are reported

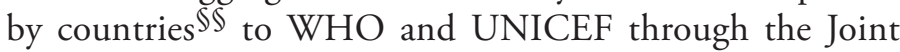
Reporting Form (JRF). Genotype data are reported to the WHO Measles Nucleotide Surveillance (MeaNS) and Rubella Nucleotide Surveillance (RubeNS) databases (5). According to JRF data, the number of specimens tested annually for measles immunoglobulin M (IgM) increased 51\% during 2010-2014,

\footnotetext{
** A suspected case that was investigated and discarded, either through negative results of adequate laboratory testing for measles or rubella or by an epidemiological link to a laboratory-confirmed case of another disease.

${ }^{\dagger} \dagger$ http://apps.who.int/immunization_monitoring/globalsummary/timeseries/ tsincidencemeasles.html.

$\$ \$$ Countries without case-based measles surveillance in 2015 were Djibouti, India, Mauritius, Sao Tome and Principe, Seychelles, and Somalia.
} 
from 171,170 to 258,339 . According to monthly reported casebased surveillance data, the number of serum specimens tested for measles IgM increased $127 \%$, from 64,864 to 146,925 during 2010-2015 (Table). The discrepancy between JRF data and monthly reported data was due in part to incomplete monthly reporting. In 2015, among the 160 countries that reported case-based surveillance data, 160,644 serum specimens were received. Of these specimens, 146,925 (91\%) were tested for measles $\operatorname{IgM}(45,674$ [31\%] positive), and 112,461 (70\%) were also tested for rubella $\operatorname{IgM}(13,601[12 \%]$ positive).

To support virologic surveillance, WHO established standard protocols for monitoring global genotype distribution and tracking transmission of measles and rubella viruses $(6)$. During 2010-2015, 27,023 measles virus sequences were submitted to MeaNS and 809 rubella virus sequences were submitted to RubeNS (Table). During 2010-2015, seven of the 24 recognized measles virus genotypes 9 and five of the 13 recognized rubella virus genotypes*** were detected (Figures 1 and 2).

\footnotetext{
I9 Sequences were for the 450-nucleotide carboxy-terminal of the nucleocapsid gene in the measles virus genome. Data as of March 28, 2016 available from the Measles Nucleotide Surveillance (MeaNS) database at http://www.who-measles.org/.

*** A 739-nucleotide fragment (nucleotides 8,731-9,469) in the E1 gene of rubella viruses is the standard sequence window in the rubella virus genome. Data as of March 28, 2016 available from the Rubella Nucleotide Surveillance (RubeNS) database at http://www.who-rubella.org/.
}

In 2014, a procedure was introduced to MeaNS that designates eligible measles sequences from contemporary outbreak strains as named strains. This designation makes it possible to monitor the global transmission patterns of defined lineages of measles virus ( 6 ).

\section{Accreditation and Quality Assurance}

An annual accreditation and proficiency testing program has been developed to ensure high quality standardized laboratory testing and to monitor the performance of serologic testing in the network laboratories (Table). Approximately 95\% of participating laboratories passed annually. A GMRLN external quality assurance program for molecular testing was established in 2014 and is coordinated on behalf of WHO by the global specialized laboratory at CDC and at the INSTAND e.V. in Berlin. From 2014 to 2015, the number of laboratories that participated in the molecular proficiency testing program increased from 22 to 90, and all but one passed in 2015 .

\section{Quality of Laboratory-Based Surveillance}

Performance indicators for collection of samples for case confirmation and timeliness of reporting of laboratory results are being met by most laboratories. However, in 2015,

TABLE. Summary of serologic testing, quality control, and viral sequence submission for the Global Measles and Rubella Laboratory Network (GMRLN), 2010-2015

\begin{tabular}{|c|c|c|c|c|c|c|}
\hline \multirow[b]{2}{*}{ Characteristic } & \multicolumn{6}{|c|}{ Year } \\
\hline & 2010 & 2011 & 2012 & 2013 & 2014 & 2015 \\
\hline \multicolumn{7}{|c|}{ No. of serum samples tested for measles IgM } \\
\hline \multicolumn{7}{|c|}{ Data source* } \\
\hline JRF & 171,170 & 152,810 & 148,177 & 197,469 & 258,339 & NA \\
\hline Monthly data & 64,864 & 85,953 & 122,719 & 160,611 & 161,115 & 146,925 \\
\hline \multicolumn{7}{|c|}{$\begin{array}{l}\text { No. of GMRLN laboratories participating in the WHO serologic proficiency test panel }{ }^{\dagger} \\
\text { WHO region }\end{array}$} \\
\hline African & 41 & 35 & 33 & 35 & 37 & 29 \\
\hline Americas & 24 & 24 & 24 & 23 & 24 & 8 \\
\hline Eastern Mediterranean & 21 & 18 & 21 & 22 & 21 & 19 \\
\hline European & 69 & 70 & 67 & 71 & 71 & 60 \\
\hline South-East Asia & 20 & 13 & 21 & 23 & 24 & 16 \\
\hline Western Pacific & 48 & 51 & 51 & 53 & 53 & 53 \\
\hline Total & 223 & 211 & 217 & 227 & 230 & 185 \\
\hline \multicolumn{7}{|c|}{ No. of sequences submitted to the GMRLN databases annually $\S$} \\
\hline Measles & 4,227 & 5,722 & 2,847 & 2,379 & 7,260 & 4,588 \\
\hline Rubella & 67 & 143 & 110 & 38 & 147 & 304 \\
\hline
\end{tabular}

Abbreviations: IgM = immunoglobulin M; JRF = Joint Reporting Form; WHO = World Health Organization.

* The annual number of serum specimens tested by the WHO GMRLN for measles IgM by year during 2010-2015 as reported through the WHO-UNICEF JRF and through the monthly aggregate laboratory data reported to WHO. WHO and UNICEF jointly collect information through a standard questionnaire, the JRF, sent to all member states. Information collected in the JRF includes estimates of national immunization coverage, reported cases of vaccine-preventable diseases, immunization schedules, and indicators of immunization system performances (http://www.who.int/immunization/monitoring_surveillance/routine/reporting/reporting/en). JRF data are available at http://www.who.int/immunization_monitoring/data/data_subject/en/.Monthly reported data are available at http://apps.who.int/immunization_ monitoring/globalsummary/timeseries/tsincidencemeasles.html. The discrepancy between JRF data and monthly reported data was due in part to incomplete monthly reporting; for example, in 2014, 37 countries did not report monthly laboratory data. JRF data for 2015 were not available at the time of press.

${ }^{\dagger}$ An annual accreditation and proficiency testing program to ensure high quality standardized laboratory testing and to monitor the testing performance of the network laboratories. An annual serologic proficiency test panel is administered by the Victorian Infectious Disease Reference Laboratory in Melbourne, Australia. Data for 2015 were incomplete at time of press.

$\S$ Data available as of March 28, 2016 from the Measles Nucleotide Surveillance database (http://www.who-measles.org/) and the Rubella Nucleotide Surveillance database (http://www.who-rubella.org/). 


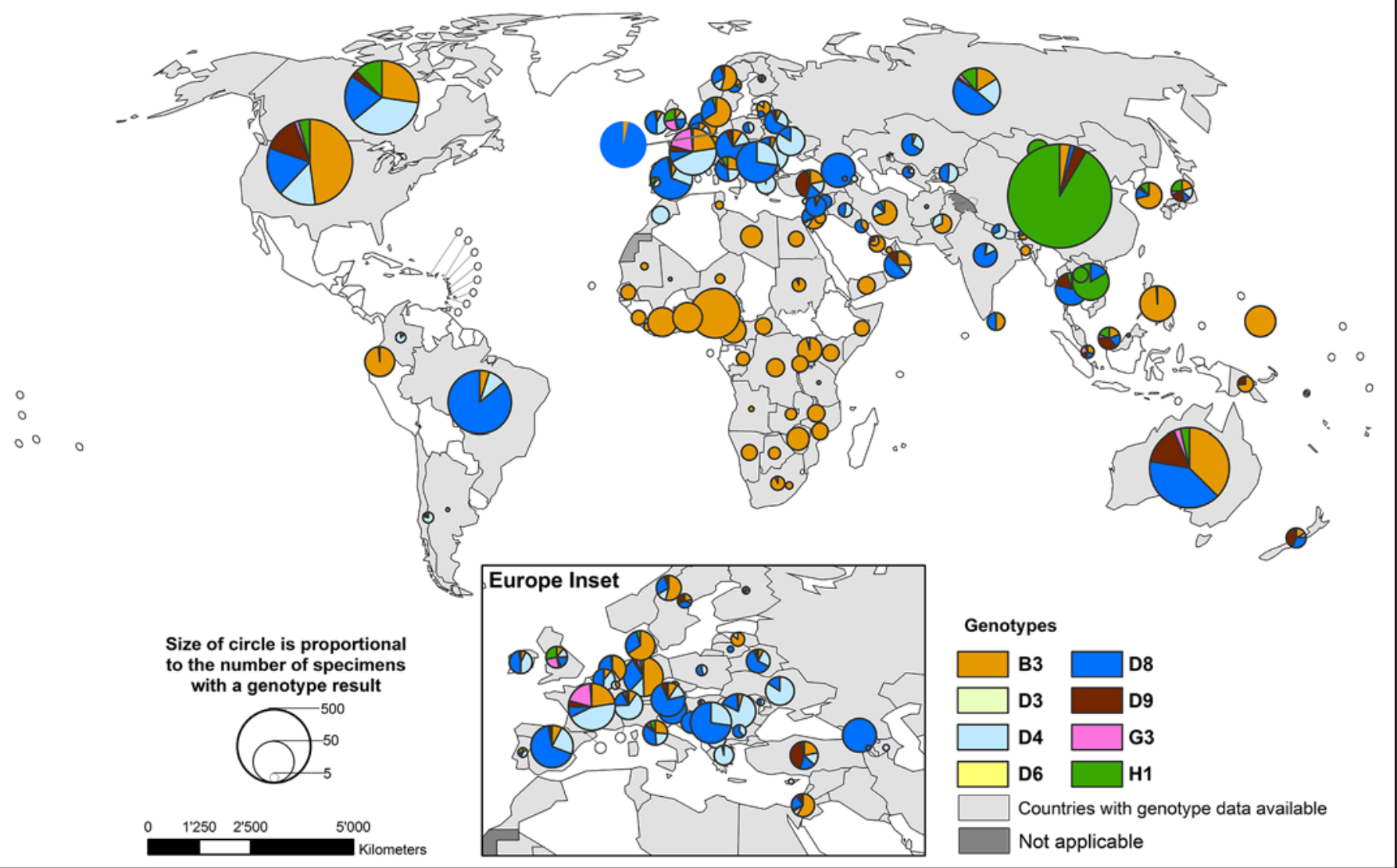

* The size of the pie chart is proportional to the number of sequences reported by the country during 2010-2015, except for China, where the size is reduced tenfold.

${ }^{\dagger}$ Measles viral sequences were for the 450-nucleotide carboxy-terminal of the nucleocapsid gene. Data available as of March 28, 2016 from the Measles Nucleotide Surveillance database (http://www.who-measles.org/).

62 countries $^{\dagger \dagger}(32 \%)$ were not able to report rates of discarded cases, and another $46(24 \%)$ reported less than one discarded case per 100,000 population. Twenty $(10 \%)$ countries reported discarded cases of one to two per 100,000 population, and only 67 (34\%) countries achieved the target discarded rate of two or more per 100,000 population.

To verify the interruption of endemic measles or rubella virus transmission in a country, detailed epidemiologic case investigations and collection of specimens for virologic sequence analysis are required. Although WHO recommends that genotype information should be obtained from $\geq 80 \%$ of transmission chains, and baseline virologic surveillance should be conducted in all countries (4), the number of countries reporting genotype data is much lower than the number of countries reporting laboratory-confirmed cases. In 2015,

\footnotetext{
it† Several countries in the European Region, American Region, and Western Pacific Region were unable to report discarded rates because most routine testing is performed by private laboratories.
}

among the 116 countries that reported laboratory-confirmed measles cases, $56(48 \%)$ reported measles virus genotype information, and among the 106 countries that reported laboratory-confirmed rubella cases, only $11(10 \%)$ reported rubella genotype information (Figures 1 and 2).

\section{Discussion}

The capacity of the GMRLN to support elimination efforts substantially increased during 2010-2015; all laboratories now follow standard testing protocols and participate in a rigorous quality control program. In addition, all regional laboratories and many national laboratories have established molecular testing, including reverse transcription-polymerase chain reaction (RT-PCR) and sequence analysis. The global specialized laboratories and regional reference laboratories conduct periodic training workshops and convene annual meetings to review progress and develop recommendations to improve laboratory-based surveillance. In many countries, GMRLN laboratories provide a platform for strengthening 


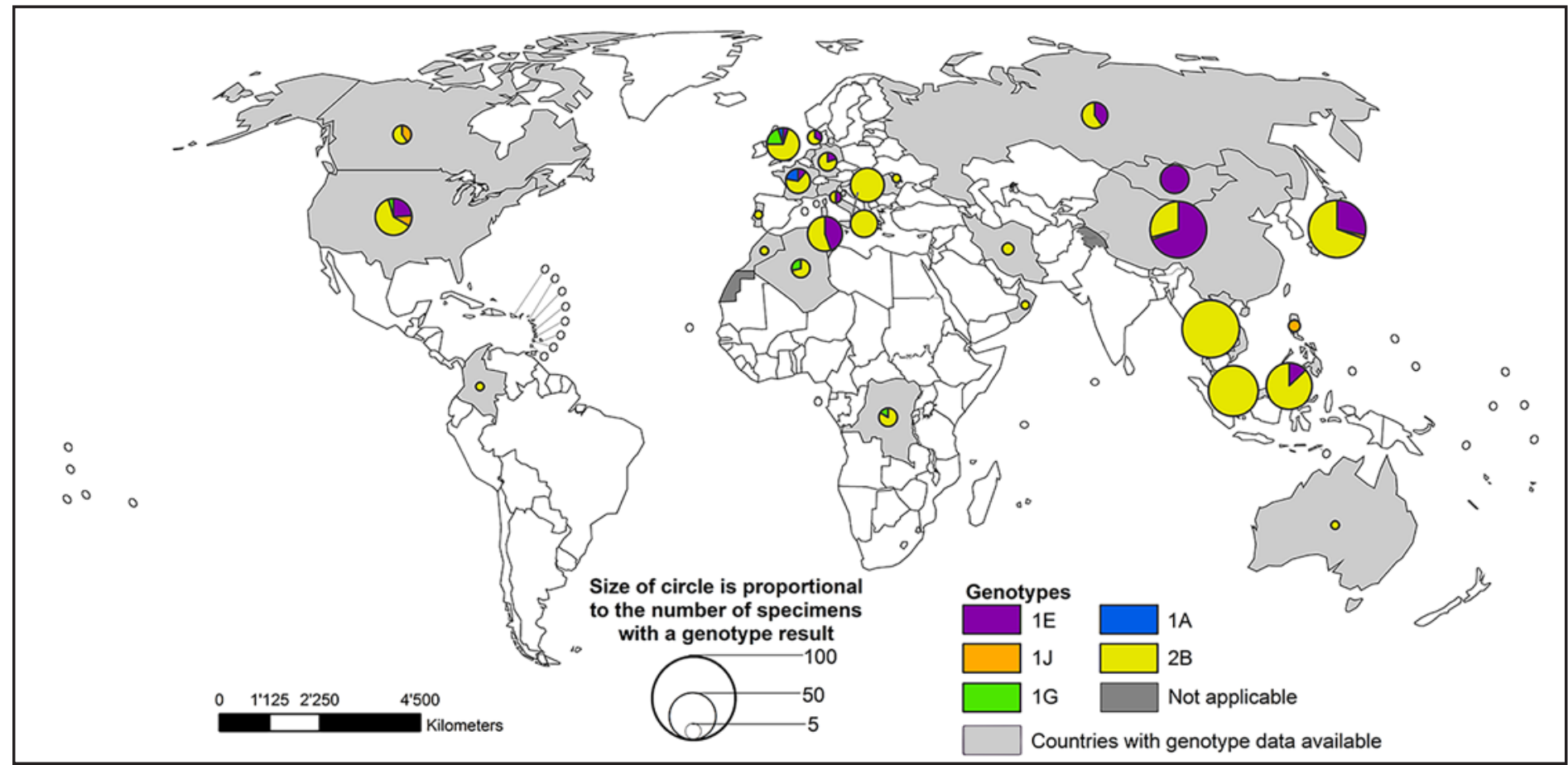

* The size of the pie chart is proportional to the number of sequences reported by the country during 2010-2015.

† A 739-nucleotide fragment (nucleotides 8,731-9,469) in the E1 gene of rubella viruses is the standard sequence window in the rubella virus genome. Data available as of March 28, 2016 from the Rubella Nucleotide Surveillance database (http://www.who-rubella.org/).

overall laboratory capacity and surveillance for other VPDs, including yellow fever and Japanese encephalitis, and support detection and response activities during public health emergencies, such as those caused by the Ebola, chikungunya, dengue, and Zika viruses. Further integration of surveillance for other VPDs, including rotavirus diarrhea, is feasible and will help sustain the investments made in establishing and building GMRLN capacity.

A continuing challenge to the GMRLN is a long-standing shortage of human and financial resources. The workload will increase as the GAVP regional measles and rubella elimination targets draw near. The laboratory network will need to expand to meet the demand for high quality laboratory data to support verification of elimination, particularly in the South-East Asia Region, with two new national laboratories to be nominated in Myanmar and Nepal and at least 20 new subnational laboratories planned for Indonesia, India, and Thailand. To address the challenge of ongoing training needs related to personnel turnover and network expansion, GMRLN conducts workshops and intensive onsite training activities in all regions.

To enhance measles and rubella elimination efforts, the GMRLN continues to develop and evaluate novel technologies, including molecular methods such as RT-PCR to confirm cases. Evaluations of high throughput serologic assays for more efficient assessments of population immunity and point-of-care assays for rapid case confirmation in remote areas have demonstrated promising results as potential new tools. Advanced molecular techniques, including the use of next-generation sequencing, should improve the resolution of molecular epidemiologic studies.

Efforts will increasingly focus on achieving regional measles and rubella elimination goals as polio eradication approaches (7). Polio legacy planning has begun to transition polio assets to strengthen routine immunization services and measles and rubella elimination efforts, while maintaining the essential polio functions of containment and surveillance (8). In many countries, Global Polio Laboratory Network and GMRLN laboratories are already located in the same institution and share personnel, infrastructure, quality control programs, technical training, and biosafety/biosecurity procedures. An advanced state-of-the-art global laboratory network providing real-time disease surveillance has been the backbone of the polio eradication program. Therefore, ensuring the sustainability and strengthening of the GMLRN should be designated as a high priority for polio legacy planning and transitioning of polio assets. 


\section{Summary}

What is already known about this topic?

Laboratory confirmation of suspected cases of measles, rubella, and congenital rubella syndrome is an essential component of surveillance for these diseases. The Global Measles and Rubella Laboratory Network (GMRLN), initiated in 2000, has made substantial progress in providing high quality laboratory surveillance needed to verify achievement of measles and rubella elimination targets.

What is added by this report?

The GMRLN is the largest globally coordinated laboratory network, with 703 laboratories supporting surveillance in 191 countries. During 2010-2015, >700,000 serum specimens were tested, and $>20,000$ viral sequences were reported globally. During the past year, the number of laboratories that participated in molecular proficiency testing increased from 22 to 90 . Performance indicators for collection of samples for case confirmation and timeliness of reporting of laboratory results are being met by most laboratories.

What are the implications for public health practice?

High quality surveillance is only possible if suspected measles, rubella, and congenital rubella syndrome cases can be quickly confirmed by the laboratory. The GMRLN's focus on standardization and quality control ensures that public health workers can rely on timely and accurate results. Building and maintaining this advanced state-of-the-art global laboratory network, capable of providing real-time disease surveillance, gives support to measles and rubella elimination efforts and surveillance for other vaccine-preventable diseases and for emerging pathogens of public health concern.

\footnotetext{
${ }^{1}$ Department of Immunization, Vaccines, and Biologicals, World Health Organization (WHO); ${ }^{2}$ Division of Viral Diseases, National Center for Immunization and Respiratory Diseases, CDC; ${ }^{3}$ Virus Reference Department, Public Health England, Colindale-London, United Kingdom; ${ }^{4}$ National Institute of Infectious Diseases, Tokyo, Japan ${ }^{5}$ Comprehensive Family Immunization, WHO Regional Office for the Americas, Pan American Health Organization, Washington, D.C.; ${ }^{6}$ Vaccine-preventable Diseases and Immunization, WHO Regional Office for Europe, Copenhagen, Denmark; ${ }^{7}$ Accelerated Immunisations Initiatives Program, WHO Regional Office for Africa, Intercountry support team West and Central Africa, Ouagadougou, Burkina Faso; ${ }^{8}$ Accelerated Immunisations Initiatives Program, WHO Regional Office for Africa, Intercountry support team East and Southern Africa, Harare,

Zimbabwe; ${ }^{9}$ Vaccine-preventable Diseases and Immunization, WHO Regional Office for the Eastern Mediterranean Region, Cairo, Egypt; ${ }^{10}$ Immunization and Vaccine Development, WHO Regional Office for Southeast Asia, New Delhi, India; ${ }^{11}$ Expanded Programme on Immunization, WHO Regional Office for Western Pacific, Manila, Philippines; ${ }^{12}$ Global Immunization Division, Center for Global Health, CDC.

Corresponding author: James L. Goodson, JGoodson@cdc.gov, 404-639-8170.
}

\section{References}

1. Perry RT, Murray JS, Gacic-Dobo M, et al. Progress toward regional measles elimination-worldwide, 2000-2014. MMWR Morb Mortal Wkly Rep 2015;64:1246-51.

2. World Health Organization. Global measles and rubella strategic plan, 2012-2020. Geneva, Switzerland: World Health Organization; 2012. http://www.who.int/immunization/documents/control/ ISBN_978_92_4_150339_6/en/

3. Featherstone D, Brown D, Sanders R. Development of the Global Measles Laboratory Network. J Infect Dis 2003;187(Suppl 1):S264-9. http:// dx.doi.org/10.1086/368054

4. World Health Organization. Framework for verifying elimination of measles and rubella. Wkly Epidemiol Rec 2013;88:89-99.

5. Rota PA, Brown K, Mankertz A, et al. Global distribution of measles genotypes and measles molecular epidemiology. J Infect Dis 2011;204(Suppl 1):S514-23. http://dx.doi.org/10.1093/infdis/jir118

6. World Health Organization. Genetic diversity of wild-type measles viruses and the global measles nucleotide surveillance database (MeaNS). Wkly Epidemiol Rec 2015;90:373-80.

7. World Health Organization. Meeting of the International Task Force for Disease Eradication, November 2015. Wkly Epidemiol Rec 2016;91:61-71.

8. Cochi SL, Freeman A, Guirguis S, Jafari H, Aylward B. Global polio eradication initiative: lessons learned and legacy. J Infect Dis 2014;210(Suppl 1):S540-6. http://dx.doi.org/10.1093/infdis/jiu345 